\title{
ON THE LERAY ISOMORPHISM THEOREM
}

\section{BARRETT O'NEILL}

The theorem to which we refer is Théorème 4 in Exposé XIX, of [1]. In view of its wide usefulness, we propose to generalize this result somewhat, as follows:

Theorem. Let $\phi: A \rightarrow \bar{A}$ be a differential graded homomorphism of differential graded sheaves (operators of degree +1) on a paracompact space $X$. Suppose that $n$ is an integer and

(1) $\phi: H^{p}\left(X, H^{k} A\right) \rightarrow H^{p}\left(X, H^{k} \bar{A}\right)$ is onto if $p+k=n$ and univalent if $p+k=n+1$;

(2) $\phi: H\left(H^{+}(X, A)\right) \rightarrow H\left(H^{+}(X, \bar{A})\right)$ is an isomorphism;

(3) the degrees of $A$ and $\bar{A}$ are bounded below, or $X$ has finite (cohomology) dimension;

then $\phi: H^{n}(\Gamma A) \rightarrow H^{n}(\Gamma \bar{A})$ is onto, and $\phi: H^{n+1}(\Gamma A) \rightarrow H^{n+1}(\Gamma \bar{A})$ is univalent.

The following facts are easily established:

Lemma. Let $\psi: D \rightarrow \bar{D}$ be a differential graded homomorphism of differential graded groups (operators of degree -1) with induced homology homomorphism $\psi_{*}: H(D) \rightarrow H(\bar{D})$. Then

(4) $\psi_{0}$ univalent and $\psi_{1}$ onto imply $\psi_{* 0}$ univalent and $\psi_{* 1}$ onto. If, further, $D^{q}=\bar{D}^{q}=0$ for $q=-1$, then

(5) $\psi_{* 0}$ and $\psi_{1}$ onto imply $\psi_{0}$ onto;

(6) $\psi_{* 0}$ and $\psi_{1}$ univalent, and $\psi_{* 1}$ and $\psi_{2}$ onto, imply $\psi_{0}$ univalent.

Proof of the Theorem. Let $C$ be a fundamental sheaf [1] on $X$. Filter $\Gamma(A \circ C)$ by the degrees of $C$; then in the resulting spectral sequence, $E_{2}^{p k}=H^{p}\left(X, H^{k} A\right)$. (In general, if $B$ is a bigraded group, let $B^{(n)}=\sum_{p+k=n} B^{p k}$.) Thus (1) asserts that for $r=2$ we have $\phi: E_{r}^{(n)} \rightarrow \bar{E}_{r}^{(n)}$ onto, and $\phi: E_{r}^{(n+1)} \rightarrow \bar{E}_{r}^{(n+1)}$ univalent. By (4) and the properties of the sequence, the same is true for $r \geqq 2$. Thus in view of (3) we have $\phi: G^{(n)} \rightarrow \bar{G}^{(n)}$ onto and $\phi: G^{(n)} \rightarrow \bar{G}^{(n+1)}$ univalent, where $G$ is the associated bigraded group of $H=H(\Gamma(A \circ C))$ under the present filtration. Then since the filtration is bounded it can be shown (as in the proof of (9)) that

(7) $\phi: H I^{n} \rightarrow \bar{H}^{n}$ is onto and $\phi: H^{n+1} \rightarrow \bar{H}^{n+1}$ is univalent.

Now filter $\Gamma(A \circ C)$ by the degrees of $A$. Since now $E_{2}^{k p}$

Received by the editors September 15, 1957.

1 The group $H\left(H^{+}(X, A)\right)$ is the cohomology group of $H^{+}(X, A)=\sum_{p>0} H^{p}(X, A)$ derived from the operator of $A$. 
$=H^{k}\left(H^{p}(X, A)\right),(2)$ asserts that $\phi: E_{2}^{k p} \rightarrow \bar{E}_{2}^{k p}$ is an isomorphism for $p \geqq 1, k$ arbitrary. (Actually we use a good deal less than this.) By an induction using (4) we find that for $r \geqq 2, k$ arbitrary

(8) $\phi: E_{r}^{k p} \rightarrow \bar{E}_{r}^{k p}$ is univalent for $p \geqq 1$ and is an isomorphism for $p \geqq r-1$.

It follows that $\phi: G^{k p} \rightarrow \bar{G}^{k p}$ is univalent for $p \geqq 1, k$ arbitrary, where $G$ is the associated bigraded group of $H=H(\Gamma(A \circ C))$ derived from its present induced filtration $\left\{{ }^{k} H\right\}$. By (3) there is an integer $b$ such that ${ }^{b} H=H$, and thus $G$ is related to $H^{n}$ by the exact sequences:

$$
\begin{aligned}
& 0 \rightarrow{ }^{b+1} H^{n} \rightarrow H^{n} \rightarrow G^{b, n-b} \rightarrow 0 \\
& 0 \rightarrow G^{n 0} \rightarrow{ }^{n-1} H^{n} \rightarrow G^{n-1,1} \rightarrow 0 .
\end{aligned}
$$

These are mapped by $\phi$ into the corresponding sequences for $\bar{A}$, and since $\phi: H^{n} \rightarrow \bar{H}^{n}$ is onto, we find by repeated use of the general fivelemma that

(9) $\phi: G^{(n)} \rightarrow \bar{G}^{(n)}$ is onto.

We shall now show that

(10) $\phi: E_{r}^{k p} \rightarrow \bar{E}_{r}^{k p}$ is onto provided $r \geqq 2, k+p=n, 0 \leqq p \leqq r-2$.

By (5) this homomorphism will be onto if $\phi: E_{r}^{k-r, p+r-1} \rightarrow \bar{E}_{r}^{k r-r, p+r-1}$ and $\phi: H^{k p}\left(E_{r}\right) \rightarrow H^{k p}\left(\bar{E}_{r}\right)$ are onto. The former condition holds by (8) ; the latter is equivalent to $\phi: E_{r+1}^{k p} \rightarrow \bar{E}_{r+1}^{k p}$ being onto. We may repeat this reasoning, since (5) and (8) apply at each stage, concluding that for (10) to hold it suffices that $\phi: G^{k p} \rightarrow \bar{G}^{k p}$ be onto: but this is the case by (9). Since $H^{0}(X, A)=\Gamma(A)$, by setting $k=n$ and $r=2$ in (10) we obtain $\phi: H^{n}(\Gamma A) \rightarrow H^{n}(\Gamma \bar{A})$ onto, half of our required result.

It remains to prove that $\phi: H^{n+1}(\Gamma A) \rightarrow H^{n+1}(\Gamma \bar{A})$, or equivalently $\phi: E_{2}^{n+1,0} \rightarrow \bar{E}_{2}^{n+1,0}$, is univalent. By (6) this is true provided $\phi: E_{3}^{n+1,0}$ $\rightarrow \bar{E}_{3}^{n+1,0}$ and $\phi: E_{2}^{n-1,1} \rightarrow \bar{E}_{2}^{n-1,1}$ are univalent, and $\phi: E_{3}^{n-1,1} \rightarrow \bar{E}_{3}^{n-1,1}$ and $\phi: E_{2}^{n-3,2} \rightarrow \bar{E}_{2}^{n-3,2}$ are onto. The second and fourth of these conditions hold by (8), the third by (10). We may apply the same reasoning to the first condition and continue by an induction to show that it suffices for $\phi: G^{n+1,0} \rightarrow \bar{G}^{n+1,0}$ to be univalent. But this homomorphism is a restriction of $\phi: H^{n+1} \rightarrow \bar{H}^{n+1}$, the univalence of which was established in (7).

Corollary 1 (notation as above). If $\phi: H(X, H A) \rightarrow H(X, H \bar{A})$ and $\phi: H\left(H^{+}(X, A)\right) \rightarrow H\left(H^{+}(X, \bar{A})\right)$ are isomorphisms, then so is $\phi: H(\Gamma A) \rightarrow H(\Gamma \bar{A})$. 
CoRollary 2. If $\phi: H^{k}(A) \rightarrow H^{k}(\bar{A})$ is an isomorphism for $k \leqq n$ and univalent for $k=n+1$, and $\phi: H\left(H^{+}(X, A)\right) \rightarrow H\left(H^{+}(X, \bar{A})\right)$ is an isomorphism, then $\phi: H^{k}(\Gamma A) \rightarrow H^{k}(\Gamma \bar{A})$ is also an isomorphism for $k \leqq n$ and univalent for $k=n+1$.

\section{REFERENCE}

1. H. Cartan, Seminaire de topologie algébrique, E.N.S., 1950-1951.

University of California, Los Angeles

\section{ONE-PARAMETER TRANSFORMATION GROUPS IN THE PLANE}

PAUL S. MOSTERT

Very little is known about the action of a one-parameter group $R$ on two-space except when all orbits are circles, in which case the action is completely known [1]. In a forthcoming paper, A. Beck proves that any closed set can act as the set of fixed points for $R$. Hence, a very general description appears to be hopeless. However, here, we are able to prove the following result.

Theorem. Let $E$ be the plane and $R$ the real line acting on $E$ as a group of transformations without fixed points (i.e., no point is left fixed by all of $R$ ). If $E / R$ is Hausdorff, then $E$ is fibred as a direct product of $R$ and a cross sectioning line. Thus, $R$ is equivalent to a group of translations.

Proof. Let $x \in E$. Since $x$ is not fixed under $R$, there is a closed interval $[-a, a]=T$ about 0 in $R$, and an arc $C \subset E, x \in C$ but not an end point of $C$, such that $T^{2}(C)$ is a compact neighborhood of $x$ and the mapping $(t, c) \rightarrow t(c)$ is one to one from $T^{2} \times C \rightarrow T^{2}(C)$. That is, $C$ is a local cross section to the local orbits of $T^{2}[1]$. We shall show that $C$ is a local cross section for the orbits of $R$.

Suppose, on the contrary, that for some $z \in C$, there is an $r>a$ such that $r(z) \in T(C)$. Let $b$ be the greatest lower bound of such numbers. Then $b(z) \in-a(C)$, for if not, say $b(z)=t(c), t \in T, c \in C$, and $t>-a$, then there is a $t^{\prime},-a<t^{\prime}<0$, such that $t+t^{\prime}>-a$. Hence $\left(b+t^{\prime}\right)(z)=\left(t+t^{\prime}\right)(c) \in T(C)$. But $t^{\prime}<0$ so that $b+t^{\prime}<b$. By the choice of $b$, this implies $b+t^{\prime}<a$. Since this implies $b+t^{\prime \prime}<a$ for

Presented to the Society, April 19, 1957; received by the editors December 21, 1956. 